\title{
Integrar la enseñanza pregrado en el hospital
}

\author{
J. Martínez-Hernández
}

$\therefore$ nosotros no nos limitamos a preparar médicos. No producimos mercachifles; formamos hombres cultos. Nuestros alumnos aprenden teología, filosofía, matemáticas, física, astrología y jurisprudencia además de medicina. Después de graduarse como intelectuales completos, pueden elegir su carrera en la enseñanza, la medicina o el derecho.'

(Palabras del vicerrector del Bimaristan de Ispahán a Reb Jesse ben Benjamín. Noah Gordon: El médico)

\section{Introducción}

La presente exposición parte de dos convicciones, la primera es que la medicina se aprende a pie de enfermo, con buenos maestros y una buena metodología de aprendizaje; la segunda, que el hospital universitario como parte de la universidad es responsable de la formación de los médicos.

En esencia, el aprendizaje de la medicina ha evolucionado poco con el tiempo. De siempre el aprendiz debe acudir donde se realiza la mejor medicina de su tiempo. Lo que sí ha cambiado revolucionariamente es la medicina misma y su complejidad, la naturaleza del conocimiento médico. Hoy precisamente, por su complejidad, es imposible tener un solo maestro y el hospital desempeña un papel central para la formación del médico.

La formación de médicos, especialistas, de otros profesionales sanitarios, la producción de conoci- miento y la actividad asistencial son los productos que un hospital universitario debe dar hoy a la sociedad, de forma que éstos actúen sinérgicamente entre sí y se complementen mutuamente. La asistencia, la docencia y la investigación son, pues, las tres grandes líneas de producto que debe generar un hospital; cada una de ellas debe tener una dirección específica y, a su vez, todas ellas, una dirección integrada e integradora.

\section{¿Qué debe aprender el médico en su formación?}

La estructura del conocimiento médico se puede descomponer en varios sumandos: por una parte, habilidades adquiridas basadas en automatismos y rutinas preprogramadas cuyo aprendizaje se realiza a través de la observación, práctica, corrección y repetición; en segundo lugar, el aprendizaje de una serie de reglas que reconozcan situaciones, que éstas se interioricen y que automáticamente, ante una situación $\mathrm{A}$, demos una respuesta $B$. Un tercer componente se basa en el conocimiento basado en el razonamiento técnico, en el que el reconocimiento de una situación y su respuesta se fundamenta en el análisis sistematizado de información, para lo que se requiere el aprendizaje de técnicas de análisis y de resolución de problemas clínicos. Existe un cuarto componente constituido por elementos inaprensibles que llamamos rol médico y que también se aprende junto a otros médicos,
Director Gerente. Hospital Universitario 12 de Octubre. Madrid, España.

E-mail jmartinezh.hdoc@salud. madrid.org 


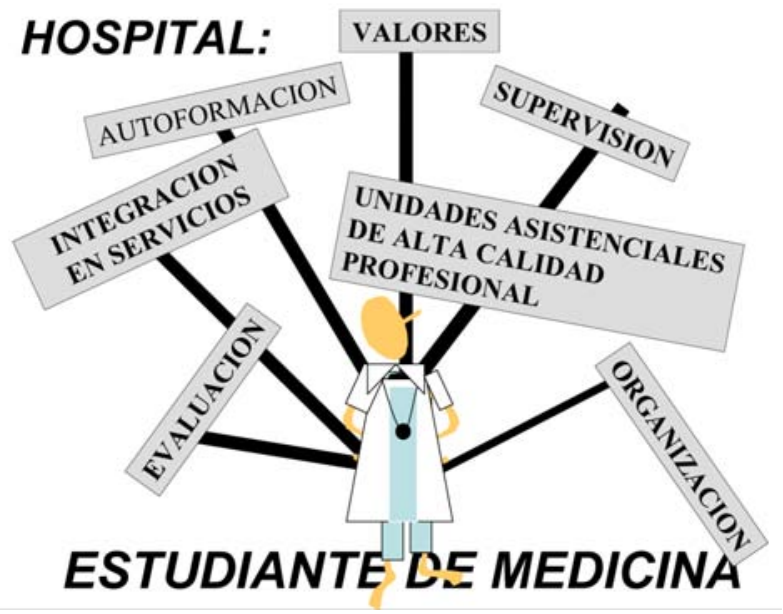

Figura 1.

como es el saber estar delante de un enfermo, el manejo de situaciones difíciles, situaciones de incertidumbre, etc.

Este conocimiento médico, que se debe de aprender en el hospital, debe estar teñido con una serie de valores que hay que inculcar justamente en esta época de la vida en la que los alumnos entran en el hospital casi como adolescentes y lo dejan como médicos adultos. En mi opinión, son dos tipos de valores los que deben interiorizar, como persona y como profesional de la medicina. Entre los primeros, creo que son destacables la honestidad, la tolerancia a la par que carácter firme según las situaciones, la responsabilidad, la capacidad crítica, la curiosidad, el interés por el entorno y por la cultura, y la integración en el medio social donde se encuentra. Entre los valores profesionales, la necesidad del aprendizaje continuo, la adquisición y perfeccionamiento de las habilidades técnicas, el interés por el conocimiento científico, la autoformación, la capacidad de comunicación y exposición, el calor humano, el tacto, la simpatía, la capacidad de comprensión, el interés por el enfermo y, en definitiva, la profesionalidad.

Es obvio que para que un estudiante pueda llegar a interiorizar estos valores, el hospital que le acoge también debe poseer otra serie de valores que alimente a aquéllos. En primer lugar el hospital debe ser una institución; entendemos por tal una organización que contribuye a vertebrar la sociedad, y más en una sociedad como la nuestra donde nuestros centros redistribuyen la riqueza y actúan como elemento equilibrador. Los hospitales deben tener también muy claro cuál es su razón de ser: mejorar la salud individual y colectiva de los ciudadanos. Debe existir una preocupación real por el paciente al igual que por el avance científico y la innovación tanto en las incorporaciones diagnósticas y terapéuticas como en las que se refieren a organización. Debe fermentar el trabajo en grupo y la capacidad de autocrítica, así como la difusión del conocimiento.

\section{¿Cómo debe ser el aprendizaje?}

El alumno de medicina debe integrarse en los diferentes servicios donde rota, y aprender la medicina con la práctica y su relación con el enfermo. En este sentido la calidad del servicio médico es un determinante importante de su calidad docente. Los servicios deben disponer de normas de funcionamiento en todas sus unidades en las que se especifique el papel de cada cual. Los principales procedimientos, técnicas, enfermedades, etc., deben estar protocolizados o incluidos en guías de práctica clínica al igual que deben estar organizadas las diferentes sesiones clínicas, bibliográficas, de revisión de casos, etc.

El papel del estudiante de medicina también debe estar definido, de manera que debe tener asignados enfermos de los que ha de conocer 


\section{Docencia médica en el Hospital.}

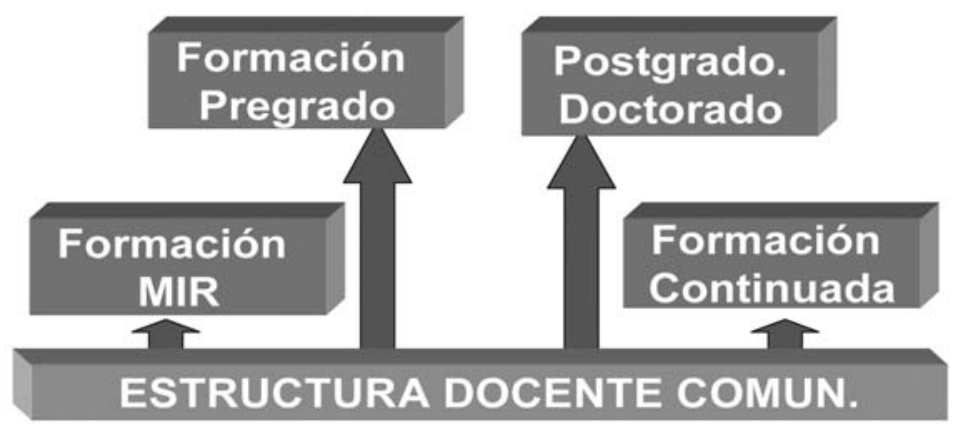

Figura 2.

sus procesos, realizar la historia clínica, la exploración física y la propuesta de exploraciones complementarias, todo lo cual debe ser revisado y corregido por el responsable médico que tenga asignado el estudiante. A diferencia del médico residente que va adquiriendo responsabilidades crecientes sobre los pacientes según transcurre su año de residencia, aunque siga siendo supervisado, el estudiante no debe tener en ningún momento responsabilidades asistenciales, lo que no significa que no deba desempeñar un papel en el servicio médico, aunque siempre supervisado.

El aprendizaje del estudiante de medicina a través de los diferentes cursos también debe estar programado de forma que, tras cada rotación por un servicio clínico, debe tener definidas las competencias que debe adquirir durante ese período de tiempo. Estas competencias no sólo se limitan al conocimiento médico, al desarrollo de habilidades o competencias en el reconocimiento y al uso de los medios de diagnóstico y tratamiento, sino también debe desarrollar competencias de comunicación con los pacientes y de interrelación con el resto de personal sanitario y no sanitario de su entorno.

Todo lo anterior es básico para el ejercicio de la medicina, pero debe completarse con otros conocimientos y actitudes que el hospital ha de ofrecer al estudiante y que también ha de adquirir necesariamente, como el aprendizaje del manejo de la información y la búsqueda bibliográfica, la iniciación a la investigación, las res- ponsabilidades éticas y legales que tendrá que asumir como médico, la importancia económica de sus decisiones, la importancia de saber idiomas, la seguridad clínica y los riesgos a que podemos someter a los pacientes, los sistemas de evaluación de la calidad asistencial y sobre todo la capacidad de autocrítica y autoaprendizaje.

Además de programado, el aprendizaje del estudiante debe ser continuamente supervisado. Para ello es necesario que en el servicio tenga un referente que le aclare dudas, le oriente, le corrija y del que aprenda la actitud que hay que mantener en situaciones difíciles o de incertidumbre. Habitualmente, en los hospitales suele existir una supervisión jerárquica: al estudiante le supervisa el residente, a éste el adjunto, el profesor, etc., lo cual no debe obviar la figura de un médico del personal referente para el estudiante (Fig. 1).

Para finalizar, la enseñanza debe ser evaluada y se ha de comprobar que, al acabar la carrera, el estudiante ha adquirido las diferentes competencias que fueron inicialmente definidas, con lo cual tendremos la seguridad de que hemos formado a un profesional que podrá llamarse, con propiedad, médico.

\section{El hospital universitario}

Para que todo lo anterior sea posible, el hospital donde el estudiante realiza sus rotaciones debe 
ASISTENCIA, DOCENCIA E INVESTIGACIÓN.

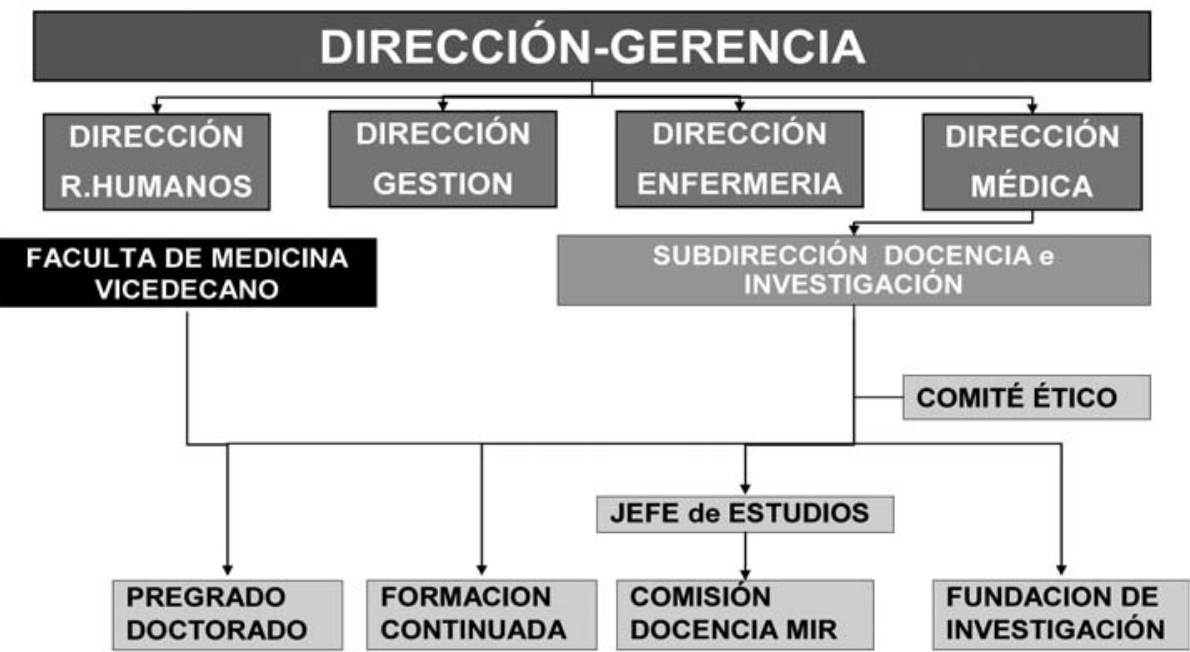

Figura 3.

tener un ambiente y una estructura docente junto a la estructura asistencial y a la estructura investigadora. Respecto a la organización docente, las diferentes estructuras hospitalarias asignadas a este fin actúan sinérgicamente entre sí, por lo que deben utilizarse para toda la docencia que se realiza en el hospital (Fig. 2).

Pero, asimismo, la estructura docente del hospital debe funcionar de forma integrada con el resto de estructuras, para lo cual debe dotarse de un responsable directivo que, además de formar parte de la comisión de dirección del centro, exponga y proponga los programas y necesidades docentes como de cualquier otra área del hospital (Fig. 3).

Con la metodología adecuada, junto al enfermo, con buenos maestros y un hospital que haya desarrollado un ambiente docente y or- ganizado tendremos todos los elementos necesarios para la buena formación de los futuros médicos.

\section{Bibliografía}

1. Friedman M. Cómo curar la sanidad. Revista de Humanidades Médicas 2003; 1: 80-112.

2. Martínez-Hernández J. Funciones y estructura de una unidad docente desde el punto de vista de la gerencia. Jornada de Comisiones Docentes y asesoras de la Comunidad de Madrid. Hospital Ramón y Cajal. Madrid, 13 de diciembre de 2001.

3. Rasmussen BK, Jensen R. La estructura del conocimiento médico. JAMA 1994; 272: 1651. 